\title{
Trabajo en equipo y Liderazgo, competencia clave para la empleabilidad de los egresados de FADE
}

\author{
Perello-Marin, M Rosario $^{a}$; Herrero-Blasco, Aurelio ${ }^{b}$ y Ribes-Giner, Gabriela ${ }^{c}$ \\ ${ }^{a}$ Departamento de Organización de empresas (Universitat Politècnica de València, España), rperell@upvnet.upv.es, \\ bepartamento de Organización de empresas (Universitat Politècnica de València, España), \\ aurelio.herrero@doe.upv.es, 'Departamento de Organización de empresas (Universitat Politècnica de València, \\ España),gabrigi@omp.upv.es.
}

\begin{abstract}
The main goal of this paper is the study of the transversal competence: teamwork ad leadership in human resources management course, in fourth year of Business administration degree, as control point for this transversal competence. The main objective is the implementation, development, control and evaluation of this competence. To the end a rubric of this competence has been used. The evidences have been gather from teamwork, learning agreement, team portfolio, hangouts, and team minutes. Considering all of them, a reasonable criterion could be reached to grade different levels of progress of the student with respect to the analysed competence.
\end{abstract}

Keywords: Competences, training, methodology, assessment, teamwork, leadership, learning agreement, team portfolio. .

\section{Resumen}

El objeto de este trabajo es el estudio de la competencia transversal: Trabajo en equipo y liderazgo en la asignatura Dirección de Recursos Humanos, de cuarto curso de Administración y Dirección de Empresas, como punto de control de esta competencia transversal. El principal objetivo es la implantación, desarrollo, control y evaluación de dicha competencia. Se parte del uso de una rúbrica de evaluación como herramienta principal. Para su implementación se emplean las evidencias procedentes de los equipos de trabajo, el contrato de aprendizaje, el portfolio, entregables, las actas del equipo y las notas del profesor/a. Con todas ellas se obtiene un criterio razonable para calificar los distintos niveles de progreso del alumno/a respecto a la gestión de su tiempo relacionado con la materia.

Palabras clave: Competencias, formación, metodología, evaluación, trabajo en equipo, liderazgo, contrato de aprendizaje, portafolio del equipo.. 


\section{Introducción}

Según las pautas marcadas por el Espacio Europeo de Educación superior, los programas de formación universitaria deben diseñarse teniendo en cuenta una serie de de competencias en aras de lograr una elevada empleabilidad y transferibilidad entre países (M.R. Perello-Marin, Vidal-Carreras, \& Marin-Garcia, 2016; Riebe, Roepen, Santarelli, $\&$ Marchioro, 2010). Ello refuerza el hecho de que la Educación Superior universitaria, la enseñanza no debería centrarse únicamente en la adquisición de conocimientos, sino también de habilidades, valores o actitudes que permitan afrontar situaciones con éxito o resolver problemas (Ricolfe et al, 2013).

En ese contexto, la Universitàt Politècnica de València (UPV) diseñó en 2013 un proyecto institucional sobre las competencias transversales UPV. Una iniciativa del Vicerrectorado de Estudios, Calidad y Acreditación, respaldada actualmente por el plan estratégico UPV2020. En este proyecto se definieron un total de trece competencias transversales (ICE-UPV 2018) cuya adquisición requiere un proceso evolutivo largo, que comienza con el inicio de los estudios de grado y se sigue perfeccionando al acabar la etapa universitaria durante el posterior desempeño diario de las funciones laborales de sus egresados. Cada competencia se asigna a un grupo de asignaturas en las que se trabaja de manera más directa. De ese modo, una vez finalizados los estudios universitarios en la UPV, el alumno tiene la opción de haber alcanzado un nivel alto en cada competencia, y haber sido evaluado por ello obteniendo de este modo una acreditación del nivel alcanzado. En la tabla 1 se pueden observar las 13 competencias transversales de la UPV.

Tabla 1. Competencias transversales de la UPV

\begin{tabular}{|l|l|}
\hline CT-01 Comprensión e integración & CT-08 Comunicación efectiva \\
\hline CT-02 Aplicación y pensamiento crítico & CT-09 Pensamiento crítico \\
\hline CT-03 Análisis y resolución de problemas & $\begin{array}{l}\text { CT-10 Conocimiento de problemas } \\
\text { contemporáneos }\end{array}$ \\
\hline $\begin{array}{l}\text { CT-04 Innovación, creatividad y } \\
\text { emprendimiento }\end{array}$ & CT-11 Aprendizaje permanente \\
\hline CT-05 Diseño y proyecto & CT-12 Planificación y gestión del tiempo \\
\hline CT-06 Trabajo en equipo y liderazgo & CT-13 Instrumental espedífica \\
\hline $\begin{array}{l}\text { CT-07 Responsabilidad ética, } \\
\text { medioambiental y profesional }\end{array}$ & \\
\hline
\end{tabular}

Fuente: ICE-UPV (2018).

A lo largo de este trabajo, se describe el modo de adquirir y evaluar la competencia transversal CT-06. "Trabajo en equipo y liderazgo" para el caso de alumnos de último curso de grado $\left(4^{\circ}\right)$. Dado el nivel académico de estos estudiantes, se entiende que comienzan el curso ya con un nivel de conocimiento y habilidad previa de esta competencia, dado que ha sido trabajada y evaluada ya en cursos anteriores.

Dicha competencia viene definida en el proyecto institucional como: 
"Trabajar y liderar equipos de forma efectiva para la consecución de objetivos comunes de un grupo de personas, contribuyendo al desarrollo personal y profesional de los mismos"

Cabe destacar que el trabajo en equipo es una de las habilidad más demandadas hoy en día a nivel profesional (Baker, Day, \& Salas, 2006; Becker, 2007; M. Rosario PerelloMarin, Vidal-Carreras, \& Marin-Garcia, 2016; Riebe et al., 2010). El gran cambio que han experimentado las organizaciones en los últimos años ha propiciado una manera de trabajar más colaborativa y cooperativa. Ello ha provocado una evolución desde un modo más autónomo e individual de trabajo, hacia un trabajo más colaborativo, en el que se requiere que dos o más trabajadores interactúen entre ellos para conseguir unos determinados resultados (Alcover, Gil, et Barrasa, 2004; Ellis et. al. 2005; Gruenfeld et. al., 1996; Hollenbeck et. al. 2004; Torrelles Nadal et Al 2011; Rousseau et. al. 2006). La complejidad de las organizaciones implica trabajar a través de objetivos comunes, en función de unos roles adquiridos o unas funciones predeterminadas. Asimismo, la gran complejidad del mundo laboral y la innovación generan diferentes situaciones que requieren diversidad de habilidades, altos niveles de conocimiento, respuestas rápidas y adaptabilidad. Y es a través de los equipos donde se pueden desarrollar todas estas características (Kozlowski et Ilgen, 2006). Esta competencia participativa permite aumentar la productividad, la innovación y la satisfacción en el trabajo (Rousseau et. al. 2006).

A pesar de la relevancia de la competencia "trabajo en equipo", a día de hoy aún son pocas las actividades que refuerzan su desarrollo entre los alumnos más allá del mero trabajo en grupo. Esto se agrava cuando se considera que el resultado al aplicar una estrategia varía de acuerdo a las capacidades y limitaciones de cada persona (Langa, 2013). Además, tal como indican los estudios empíricos existentes sobre este tipo de competencias en entornos universitarios, su adecuada evaluación exige una metodología específica (Marin-Garcia \& Lloret, 2008; Riebe et al., 2010; Tu \& Lu, 2005; Viles, Jaca, Campos, \& Serrano, 2012; Zou \& Ko, 2012). Y por otro lado, existen muchas desventajas y dificultades a la hora de la implementación del trabajo en equipo (Riebe et al., 2010), entre ellas cabe destacar la percepción de evaluación injusta por parte de los estudiantes (Gallegos \& Peeters, 2011; Halvorsen, A. L., Harris, L. M., Aponte Martinez, G., \& Frasier, 2015; M. Rosario Perello-Marin et al., 2016; Riebe et al., 2010), quienes rechazan trabajar con otros compañeros que tengan estilos de trabajo y/o aprendizaje diferentes a ellos.

Por lo tanto, para llevar al aula realmente este reto de manera eficiente, se importante acertar con las actividades y herramientas escogidas que nos permitan el desarrollo de esta competencia entre los alumnos al tiempo que adquieren las competencias específicas de la materia en cuestión. 


\section{Objetivos}

El principal objetivo de la presente investigación es realizar un estudio de las herramientas disponibles para la adquisición y evaluación de la competencia "Trabajo en equipo" mediante estrategias que sean efectivas, eficaces y flexibles. Los objetivos específicos son:

1. Análisizar la situación actual de la asignatura.

2. Identificar las destrezas requeridas para un eficaz trabajo en equipo

3. Diseñar las herramientas de evaluación de la competencia

\section{Metodología empleada}

Para el desarrollo de este trabajo se ha empleado una metodología de tipo cualitativa, tal y como se muestra a continuación.

En primer lugar se describe la muestra estudidada: es decir la asignatura y el alumnado correspondiente enmarcado la titulación a la que pertenece.

A continuación se ha recabado toda la información secundaria procedente de los distintos proyectos institucionales acerca de competencias transversales, así como otras fuentes externas a la UPV.

Finalmente se diseñan las herramientas que permiten documentar las evidencias que posteriormente se emplearán con la finalidad de emplear los sistemas de evaluación (rubricas) propuestos en el seno del proyecto institucional de competencias transversales de la UPV.

\section{Análisis de la situación actual}

La experiencia que describe a continuación corresponde a alumnos de $4^{\circ}$ grado de $\mathrm{ADE}$ en la Univesitat Politècnica de València. La asignatura punto de control en la cual se evalúa y acredita directamente el nivel de la competencia de trabajo en equipo en este curso, es "Dirección de Recursos Humanos". Al tratarse de una asignatura de $4^{\circ}$ curso, el nivel de dominio evaluado es el nivel 2.

Así pues, el resultado de aprendizaje esperado para un nivel 2 en esta competencia implica:

"Participar en equipos de trabajo, comprometiéndose y participando activamente en el logro de los objetivos de trabajo."

\section{Análisis de las destrezas requeridas para un eficaz trabajo en equipo}

Los indicadores propuestos para realizar el seguimiento de la adquisición de las destrezas propias de dicho nivel son: 
- Participar en la planificación de objetivos.

- Actuar para afrontar los conflictos del equipo.

- Comprometerse en la realización de la tarea colectiva.

Dichas destrezas se han de ver materializadas a lo largo de las diversas actividades incluidas en el desarrollo de la asignatura. Entre las actividades propuestas por parte del proyecto corporativo para la adquisición de la competencia, en esta asignatura concreta, se desarrollan las siguientes:

- Actividades grupales

- Estudios de caso

- Exposiciones orales

- Foros y debates

- Proyecto en equipo

Las evidencias de las actividades desarrolladas por cada equipo, se documentan en el portfolio del equipo. Dicho portfolio es una pieza fundamental para la evaluación posterior de esta competencia transversal, dado que recoge no sólo el trabajo realizado, si no también evidencias del proceso llevado a cabo para su realización.

\section{Análisis de las herramientas de evaluación de la competencia}

Existen diversos métodos de evaluación de la adquisición de esta competencia. Desde La UPV se ha definido una propuesta de rúbrica que puede observarse en la tabla 2.

Tabla 2. Rubrica para ela competencia trabajo en equipo

\begin{tabular}{|c|c|c|c|c|}
\hline \multirow{2}{*}{ INDICADORES } & \multicolumn{4}{|c|}{ DESCRIPTORES } \\
\hline & D. No alcanzado & C. En desarrollo & B. Bien /adecuado & A. Excelente/ejemplar \\
\hline $\begin{array}{l}\text { Participa en la } \\
\text { planificación de } \\
\text { objetivos }\end{array}$ & $\begin{array}{l}\text { No se implica en la } \\
\text { planificación }\end{array}$ & $\begin{array}{l}\text { Acepta la planificación } \\
\text { propuesta por los demás }\end{array}$ & $\begin{array}{l}\text { Participa activamente en } \\
\text { la planificación }\end{array}$ & $\begin{array}{l}\text { Lleva la iniciativa en la } \\
\text { planificación y fomenta la } \\
\text { colaboración }\end{array}$ \\
\hline $\begin{array}{l}\text { Actúa para afrontar los } \\
\text { conflictos del equipo }\end{array}$ & $\begin{array}{l}\text { Quiere imponer su criterio } \\
\text { y provoca conflictos }\end{array}$ & $\begin{array}{l}\text { Evita afrontar los } \\
\text { conflictos }\end{array}$ & $\begin{array}{l}\text { Escucha activamente y } \\
\text { participa en la resolución } \\
\text { del conflicto }\end{array}$ & $\begin{array}{l}\text { Consensua las opiniones } \\
\text { de los demás para } \\
\text { resolver problemas y } \\
\text { conflictos }\end{array}$ \\
\hline $\begin{array}{l}\text { Se compromete en la } \\
\text { realización de la farea } \\
\text { colectiva }\end{array}$ & $\begin{array}{l}\text { Realiza sus tareas de } \\
\text { manera individualista }\end{array}$ & $\begin{array}{l}\text { Realiza sus tareas de } \\
\text { forma coordinada }\end{array}$ & $\begin{array}{l}\text { Realiza sus tareas, pide y } \\
\text { ofrece ayuda cuando se } \\
\text { necesita }\end{array}$ & $\begin{array}{l}\text { Realiza sus tareas y las } \\
\text { comparte para identificar } \\
\text { dependencias y/o } \\
\text { sinergias }\end{array}$ \\
\hline
\end{tabular}

Fuente: ICE-UPV (2018). 
Cabe destacar que los métodos de evaluación empleados a fin de obtener información útil para el uso de la rúbrica anterior son:

- Portafolio del estudiante

- Autoevaluación del trabajo en equipo

- Evaluación entre iguales del trabajo en equipo

- Presentaciones orales con rúbricas

- Trabajo académico

- Cuestionario

De entre todas las herramientas que se emplean y como marco de referencia para la evaluación de esta competencia, es el portafolio del equipo el que mayor relevancia tiene.

Según Romero Cerezo (2008), el portafolio como recurso docente de innovación y evaluación es una herramienta relevante para la recogida de materiales y evidencias de aprendizaje. Suele emplearse como instrumento el que se recogen los trabajos o productos de los/as estudiantes relacionados con los objetivos de una determinada asignatura.

El portfolio incluye las evidencias documentadas de todas las actividades llevadas a cabo por el equipo (Autoevaluación del trabajo en equipo, Evaluación entre iguales del trabajo en equipo, Presentaciones orales con rúbricas, Trabajo académico, entre otros).

El primer documento que se desarrolla es el contrato del equipo. Este documento, si se diseña adecuadamente, permite pactar y clarificar entre los miembros del equipo, desde un principio, los criterios que se tendrán en cuenta en la evaluación del proceso y del trabajo final de cada equipo y, en particular, de cada estudiante.

Entre las numerosas ventajas que presenta el portafolio del equipo, según Bernal (2006), cabe destacar, el reparto de responsabilidades y tareas, la planificación del proceso, el cronograma, las reuniones de trabajo y acuerdos alcanzados, o la evaluación y puesta en común en clase de los distintos trabajos. Se trata de una herramienta que favorece la evaluación continua del alumno a medida que va avanzando la asignatura, dado que facilita la evidencia de cómo se van adquiriendo paulatinamente las así como el proceso del trabajo en equipo.

Con todo ello, el portafolio del equipo tiene una doble finalidad: formativa -recurso didáctico, dado que recoge las evidencias de los aspectos tratados a lo largo del curso- y estrategia evaluativa -dado que permite evaluar la progresión y resultados del equipo (Perez Perez, 2014).

La evaluación final del portafolio por parte del profesorado se realiza tras finalizar el curso, si bien, se realizan revisiones continuas o bien mediante tutoría o bien durante las sesiones de trabajo en el aula. Ello permite introducir correcciones, tanto en las actividades como en el método de trabajo, así como la existencia de un continuo feedback con el estudiante. 
Cabe destacar que no se trata de una mera colección organizada de todas las actividades llevadas a cabo en una asignatura (apuntes, trabajos en grupo, prácticas,etc.). Si bien dicho archivo contribuye a un mejor aprendizaje, ésta no es la única finalidad del portafolio. A través de esta carpeta, se espera que los/as estudiantes demuestren su nivel de aprendizaje en el sentido que ellos crean conveniente y en relación con los criteriosguía estipulados por la profesora al inicio de la asignatura.

Uno de los primeros documentos que contiene el portafolio es el contrato de equipo. A través de este contrato, los estudiantes que forman parte del equipo establecen un acuerdo que guía sus pautas de trabajo y es aprobado por el profesor.

Este contrato servirá como base posterior para la evaluación del trabajo en equipo por parte de los miembros del mismo y del profesor. Esta herramienta ayuda a reducir la percepción de injusticia de las notas obtenidas como resultado del trabajo en equipo.

Es importante resaltar la relevancia de incluir la evaluación del proceso del trabajo en equipo en el resultado final. Si solo se evalua el producto del trabajo en equipo, podríamos estar premiando grupos que trabajan de manera independiente y agregan sus aportaciones al final obteniendo buenos resultados debido al esfuerzo de parte de sus miembros, no logrando con ello una evidencia válida del trabajo en equipo propiamente (Riebe et al., 2010).

\section{Conclusiones}

Tras el análisis de la situación actual, en referencia a la competencia de trabajo en equipo, se ha mostrado la relevancia para la empleabilidad de la competencia transversal de trabajo en equipo. Y cómo a pesar de ello, no siempre se logra desarrollarla adecuadamente entre los estudiantes.

Entre las dificultades encontradas para el desarrollo y evaluación de esta competencia se muestra la baja percepción del alumno frente a las ventajas del desarrollo de la misma.

Una vez analizadas las destrezas requeridas para lograr un trabajo eficaz en equipo, se muestran herramientas que permiten potenciar y evaluar, no solo el resultado final obtenido si no el proceso que sigue el estudiante para lograrlo.

Entre las más significativas, cabe destacar el portafolio del equipo con el contrato de equipo como piedra angular del mismo.

Como líneas de trabajo futuras, se propone incluir dentro del portafolio herramientas de autoevaluación del equipo, tanto al comienzo de la asignatura, como al final a fin de poder ver cómo han evolucionado los alumnos tras cursar la asignatura e implantar las medidas propuestas. 


\section{Referencias}

ALCOVER, C. M., GIL, F., \& BARRASA, A. (2004). Team learning: Adaptation of learning activities scales in a spanish sample. [Aprendizaje de equipo: Adaptación en una muestra española de las escalas de actividades de aprendizaje] Psicothema, 16(3), 378383.

BAKER, D. P., DAY, R., \& SALAS, E. (2006). Teamwork as an Essential Component of High-Reliability Organizations. Health Services Research, 41(4p2), 1576-1598. https://doi.org/10.1111/j.1475-6773.2006.00566.x

BECKER, W. S. (2007). Field of dreams: team implementations and greenfields. Team Performance Management: An International Journal, 13(3/4), 65-89. https://doi.org/10.1108/13527590710759838

ELLIS, A.P.J., BELL, B.S., PLOYHART, R. E., HOLLENBECK, J. R., \& ILGEN, D.R. (2005). An evaluation of generic teamwork skills training with action teams: effects on cognitive and skill-based outcomes. Personnel psychology, 58, 641-672

GALLEGOS, P. J., \& PEETERS, J. M. (2011). A measure of teamwork perceptions for team-based learning. Currents in Pharmacy Teaching and Learning, 3(1), 30-35. https://doi.org/10.1016/j.cpt1.2010.10.004

GRUENFELD, D.H.; MANNIX, E.A.; WILLIAMS, K.Y.; ET NEALE, M.A. (1996) Group Composition and Decision Making: How Member Familiarity and Information Distribution Affect Process and Performance. Organizational Behavior and Human Decision Processes, 67 (1), 1-15.

HALVORSEN, A. L., HARRIS, L. M., APONTE MARTINEZ, G., \& FRASIER, A. S. (2015). Does students' heritage matter in their performance on and perceptions of historical reasoning tasks?., Journal of Curriculum Studies, 0272 (November), 1-22. https://doi.org/10.1080/00220272.2015.1092585

HOLLENBECK JR, DERUE DS, GUZZO R. (2004). Bridging the gap between I/O research and HR practice: Improving team composition, team training and team task design.Human Resource Management, 43, 353-366.

ICE-UPV. (2018). Obtenido de Proyecto de competencias transversales: https:/poliformat.upv.es/access/content/group/ESP_0 2254/PROYECTO\%20COMPE TENCIAS\%20TRANSVERSALES\%20DE\%20LA\%20UPV \%2027.07.18.pdf

KOZLOWSKI, S. W. J., \& ILGEN, D. R. (2006). Enhancing the efectiveness of work groups and teams. Psychological Science in the Public Interest, Supplement, 7(3), 77124.

MARIN-GARCIA, J. A., \& LLORET, J. (2008). Improving teamwork with university engineering students. The effect of an assessment method to prevent shirking. ... on Advances in Engineering Education, 5(1), 1-11. 
OLIVER, J. (2016). Desarrollo multinivel de la competencia transversal de trabajo en equipo. In Actas de las XXII JENUI(pp. 119-126). Universidad de Almería.

PERELLO-MARIN, M. ROSARIO, VIDAL-CARRERAS, P. I., \& MARIN-GARCIA, J. A. (2016). What Do Undergraduates Perceive About Teamwork? International Journal of Engineering Education, 22(3(A)), 1171-1181.

PÉREZ-PÉREZ, I., 2014. El trabajo en equipo mediante el uso del portafolio y las rúbricas de evaluación. Revista d'innovació i recerca en educació., 7(1), pp.56-75.

RICOLFE, C., SERAFIN, J., \& ESCRIBÁ PÉREZ, C. (2013). Análisis de la percepción de las competencias genéricas adquiridas en la Universidad. Revista de Educación, (362), 535-561.

RIEBE, L., ROEPEN, D., SANTARELLI, B., \& MARCHIORO, G. (2010). Teamwork: effectively teaching an employability skill. Education + Training, 52(6/7), 528-539. https://doi.org/10.1108/00400911011068478

ROMERO CEREZO, C. (2008). El portafolio y el trabajo de grupo: una experiencia del crédito ECTS en la formación del magisterio especialista en Educación Física. REIFOP, $11(2), 73-84$.

ROUSSEAU, V., AUBE, C., \& SAVOIE, A. (2006). Teamwork behaviors: A review and an integration of frameworks. Small Group Research, 37(5), 540-570.

TORRELLES NADAL, C., COIDURAS RODRÍGUEZ, J. L., ISUS, S., CARRERA, X., PARÍS MAÑAS, G., \& CELA, J. M. (2011). Competencia de trabajo en equipo: definición y categorización. Profesorado: revista de currículum y formación del profesorado, 2011, vol. 15, núm. 3, p. 329-344.

TU, Y., \& LU, M. (2005). Peer-and-Self Assessment to Reveal the Ranking of Each Individual's Contribution to a Group Project. Journal of Information Systems Education, 16(2), 197-205.

VILES, E., JACA, C., CAMPOS, J., \& SERRANO, N. (2012). Evaluación de la competencia de trabajo en equipo en los grados de ingeniería Assessment of teamwork skills in engineering degree course, 67-75.

ZOU, T. X. P., \& KO, E. I. (2012). Teamwork development across the curriculum for chemical engineering students in Hong Kong: Processes, outcomes and lessons learned. Education for Chemical Engineers, 7(3), e105-e117. https://doi.org/10.1016/j.ece.2012.04.003 